\title{
ISOMETRIES OF ORLICZ SPACES
}

BY G. LUMER

Communicated by Ralph Phillips, June 13, 1961

The purpose of the present note is to sketch a solution for the problem of determining the form of all isometries of any reflexive Orlicz space. ${ }^{1} \mathrm{~A}$ partial result in that direction was obtained earlier by J. Lamperti [4] (who suggested this problem to us recently). The ideas of the proof are very closely related to those used recently by the author to develop a unified and slightly extended theory (unpublished) [6] for the classical results of Banach [1], Stone [8] and Kadison [2] (see also [4]) on isometries of $C(X), L_{p}$ spaces, and $C^{*}$ algebras. The systematic use of semi-inner-product spaces, and generalized hermitians [5], plays a central role. A semi-inner-product space, is a vector space $X$ on which there is defined a (complex valued) form $[x, y]$ satisfying:

(i) Linearity in $x$,

(ii) $[x, x]>0$ if $x \neq 0$,

(iii) $|[x, y]|^{2} \leqq[x, x][y, y]$.

$X$ is then normed under $\|x\|=[x, x]^{1 / 2}$.

From now on, $X$ is a reflexive Orlicz space $[7 ; 3]$ whose unit sphere is the set $\left\{f \in X: \int \phi(|f|) \leqq 1\right\}$. It is somewhat laborious but not very difficult to show that the semi-inner-product for $X$ is given by:

$$
[f, g]=C(g) \int_{\mathbf{\Omega}} f \phi^{\prime}\left(\frac{|g|}{\|g\|}\right) \operatorname{sgn} g
$$

where

$$
\operatorname{sgn} g=\left\{\begin{array}{cc}
\frac{|g|}{g} & \text { if } g \neq 0, \\
0 & \text { if } g=0
\end{array}\right.
$$

with $C(g)=\left(\int g \phi^{\prime}(|g| /\|g\|) \operatorname{sgn} g\right)^{-1}\|g\|^{2}$, when $g$ is such that the measure of $\{\xi \in \Omega: \phi$ has no derivative at the point $|g(\xi)| /\|g\|\}$ is 0 .

A bounded hermitian operator (see [5]) satisfies by definition $[H f, f]=$ real for all $f \in X$.

Proposition 1. If $h$ is real valued and in $L_{\infty}(\Omega), H f=h f$ defines $a$ hermitian operator on $X$, and $\|H\|=\|h\|_{\infty}$.

${ }^{1}$ Actually the proof sketched below covers the Orlicz spaces over measure spaces containing no atoms. If the measure space contains atoms, further argument is needed. 
THEOREM $2 .^{2}$ If $X$ is different from $L_{2}(\Omega), H$ is a bounded hermitian on $X$, then there is a real valued $h \in L_{\infty}(\Omega)$ such that $H f=h f$ for all $f \in X$, and $\|H\|=\|h\|_{\infty}$.

SKETCH OF THE PROOF. If $u$ and $v$ are in $X$, and have disjoint supports, $\Omega_{1}$ and $\Omega_{2}$, then $\operatorname{Im}\left[H\left(e^{i \alpha} u+e^{i \beta_{v}}\right), e^{i \alpha} u+e^{i \beta_{v}}\right]=0 . \alpha, \beta$ real and arbitrary lead to.

$$
\int_{\Omega_{2}} H u \phi^{\prime}(|v| /\|v\|) \operatorname{sgn} v=\left\{\int_{\Omega_{1}} H v \phi^{\prime}(|u| /\|u\|) \operatorname{sgn} u\right\} .
$$

One applies this to $u_{2}=\alpha \chi_{\Omega_{1}}, u_{3}=\beta \chi_{\Omega_{1}}, u_{1}=(\alpha+\beta) \chi_{\Omega_{1}}$ and $v=\chi_{\Omega_{2}} /\left\|\chi_{\Omega_{2}}\right\|$, where $\chi_{\Omega}$ denotes the characteristic function of the measurable set $\Omega$. One arrives finally at:

$$
\left(\phi^{\prime}\left(\frac{\alpha+\beta}{\lambda_{1}}\right)-\frac{\phi^{\prime}\left(\frac{1}{\lambda_{1}}\right)}{\phi^{\prime}\left(\frac{1}{\lambda_{2}}\right)} \phi^{\prime}\left(\frac{\alpha}{\lambda_{2}}\right)-\frac{\phi^{\prime}\left(\frac{1}{\lambda_{1}}\right)}{\phi^{\prime}\left(\frac{1}{\lambda_{3}}\right)} \phi^{\prime}\left(\frac{\beta}{\lambda_{3}}\right)\right) \int_{\Omega_{1}} H v=0
$$

where $\lambda_{1}=\left\|u_{1}+u_{2}+v\right\|, \lambda_{2}=\left\|u_{1}+v\right\|, \lambda_{3}=\left\|u_{2}+v\right\|, \alpha, \beta>0$ arbitrary $\Omega_{1}, \Omega_{2}$ and $v$ fixed. Letting the measure of $\Omega_{1}$ tend to 0 in a convenient manner $\lambda_{1}, \lambda_{2}$ and $\lambda_{3}$ tend to $\|v\|=1$, so that either $\phi^{\prime}(\alpha+\beta)=\phi^{\prime}(\alpha)$ $+\phi^{\prime}(\beta)$ (i.e., $\phi(\alpha)=k \alpha^{2}$ and $X$ is $L_{2}(\Omega)$ ) or else $H v$ is 0 on $\Omega_{1}$. From this follows that if $f \in X$ is a step function and $\Omega_{0}$ the support of one step, $H\left(f-f\left(\Omega_{0}\right) 1\right)$ is 0 on $\Omega_{0}$, hence $H f=h f$, where $h=H 1$. The rest is immediate. From this we obtain the main theorem.

THEOREM 3. If $U$ is an isometry from $X$ onto $X$, then it is of the form $\operatorname{Uf}(\cdot)=u(\cdot) f(T \cdot)$ where $T$ is a measurable transformation in $\Omega$ and $u$ a fixed function in $X$, unless $X$ is a Hilbert space.

SKETCH OF THE PROOF. The expression $[f, g]^{\prime}=[U f, U g]$ is again a semi-inner-product on $X$, so that if $H$ is hermitian the same holds for $U H U^{-1}$. If the real-valued function $h \in L_{\infty}(\Omega)$, denote by $H_{h}$ the multiplication operation defined by $h$ (which is hermitian). $U H_{h} U^{-1}$ $=H_{\hat{h}}$, where $\|\hat{h}\|_{\infty}=\|H\|=\|h\|_{\infty}$. Since $U H_{h} U^{-1} U H_{k} U^{-1}=U H_{h k} U^{-1}$, the operation $\wedge$ is multiplicative, and step functions go into step functions. This defines $T$; the rest goes smoothly.

REMARK. The previous argument could be modified so as to hold for a form not satisfying condition (iii), if a sufficiently strong condi-

${ }^{2}$ From a letter I received recently from Dr. C. A. McCarthy, it appears that McCarthy had a proof of Theorem 2. 
tion is assumed with respect to $\phi$. The space would not be an Orlicz space, but an extension of the $L_{p}$ space for $p<1$. For the latter $L_{p}$ spaces, it is known that the isometries are as described above.

\section{REFERENCES}

1. S. Banach, Thêorie des opérations linéaires, Warsaw, Monogr. Mat., Tom 1, 1932.

2. R. V. Kadison, Isometries of operator algebras, Ann. of Math. vol. 54 (1951) pp. 325-338.

3. M. A. Krasnosel'ski and Ya. Ruticki, Convex functions and Orlicz spaces (in Russian), Moscow, Gosudarstv. Izdat. Fiz.-Mat. Lit., 1958.

4. J. Lamperti, On the isometries of certain function spaces, Pacific J. Math. vol. 8 (1958) pp. 459-466.

5. G. Lumer, Semi-inner-product spaces, Trans. Amer. Math. Soc. vol. 100 (1961) pp. $29-43$.

6. - Semi-inner-product spaces and isometries, (to be published).

7. W. Orlicz, Über eine gewisse Klasse von Räumen von Typus B, Bull. Inst. Acad. Polon. Sci. Ser. A (1932) pp. 207-220.

8. M. H. Stone, Applications of the theory of Boolean rings to general topology, Trans. Amer. Math. Soc. vol. 41 (1937) pp. 375-481.

\section{STANFORD UNIVERSITY}

\section{ON THE RECURRENCE OF SUMS OF RANDOM VARIABLES}

\section{BY K. L. CHUNG ${ }^{1}$ AND DONALD ORNSTEIN ${ }^{2}$}

Communicated by J. L. Doob, September 19, 1961

We give a very short proof of the recurrence theorem of Chung and Fuchs [1] in one and two dimensions. This new elementary proof does not detract from the old one which uses a systematic method based on the characteristic function and yields a satisfactory general criterion. But the present method, besides its brevity, also throws light on the combinatorial structure of the problem.

Let $\mathrm{N}$ denote the set of positive integers, $\mathrm{M}$ that of positive real numbers. Let $\left\{X_{n}, n \in \mathrm{N}\right\}$ be a sequence of independent, identically distributed real-valued random vectors, and let $S_{n}=\sum_{\nu=1}^{n} X_{\nu}$. The value $x$ is possible iff for every $\epsilon>0$ there exists an $n$ such that $P\left\{\left|S_{n}-x\right|<\epsilon\right\}>0$; it is recurrent iff for every $\epsilon>0, P\left\{\left|S_{n}-x\right|<\epsilon\right.$ for infinitely many $n\}=1$. It is shown in [1] that every possible value is recurrent if and only if for some $m \in M$ we have

$$
\sum_{n=1}^{\infty} P\left\{\left|S_{n}\right|<m\right\}=\infty \text {. }
$$

1 This research is supported in part by the United States Air Force Office of Scientific Research under Contract AF 49(638)-265.

${ }^{2}$ This research is supported in part by the NSF Grant 16434 . 